\title{
ON STABLE BLOCKS OF AUSLANDER-ALGEBRAS
}

\author{
BY
}

\author{
CHRISTINE RIEDTMANN
}

\begin{abstract}
The Auslander-algebra $E_{\Lambda}$ of an algebra $\Lambda$ of finite representation type is the endomorphism algebra of the direct sum $M=\oplus M_{i}$ of one copy of each indecomposable $\Lambda$-module. A stable block of $E_{.1}$ is a connected direct factor of the residue algebra of $E_{1}$ modulo the two-sided ideal generated by the projections of $M$ to the $M_{i}$ 's that are not stable under $D T r$. This paper describes the stable blocks whose quiver is a stable translation-quiver of class $A_{n}$ or $D_{n}$.
\end{abstract}

1. Introduction. Let $\Lambda$ be a finite-dimensional associative algebra over an algebraically closed field $k$, and assume $\Lambda$ has only a finite number of indecomposable modules $M_{1}, M_{2}, \ldots, M_{s}$, up to isomorphism. The endomorphism ring $E_{\Lambda}$ of the direct sum $M=\oplus M_{i}$ is called the Auslander-algebra of $\Lambda$. It is a finite-dimensional basic $k$-algebra which determines $\Lambda$ up to Morita equivalence [1], and its quiver is the Auslander-Reiten quiver $\Gamma_{\Lambda}$ of $\Lambda$ (see e.g. $[5,1.1]$ ). $\Lambda$ is called standard if $E_{\Lambda}$ is isomorphic to the residue algebra of the path algebra $k \Gamma_{\Lambda}$ modulo the ideal of mesh-relations of $\Gamma_{\Lambda}[2]$. There are examples of nonstandard algebras, although only in case $k$ has characteristic 2, and the number of nonisomorphic basic connected algebras with the same Auslander-Reiten quiver can in fact be arbitrarily large though finite [7-9].

It seems to be an impossible task to describe all connected Auslander-algebras by their quivers and relations. For one thing, not much is known about AuslanderReiten quivers. The intrinsic criterion given by Bongartz and Gabriel in [2] for a translation-quiver to be the Auslander-Reiten quiver of a representation-finite algebra $\Lambda$ is hard to verify. However, the stable part ${ }_{s} \Gamma_{\Lambda}$ of an Auslander-Reiten quiver $\Gamma_{\Lambda}$ is easy to describe. A vertex $M_{i}$ of $\Gamma_{\Lambda}$ is called stable if $M_{i}$ is isomorphic to $\tau^{r} M_{i}$ for some integer $r \neq 0$, where $\tau$ denotes the Auslander-Reiten translation of $\Gamma_{\Lambda}$, and the stable part ${ }_{s} \Gamma_{\Lambda}$ of $\Gamma_{\Lambda}$ is the full subtranslation-quiver of $\Gamma_{\Lambda}$ whose vertex set consists of the stable vertices of $\Gamma_{\Lambda}$. This stable part ${ }_{s} \Gamma_{\Lambda}$ is a disjoint union of translation-quivers of the form $\mathbf{Z} \Delta / \Pi$, where $\Delta$ is one of the Dynkin-graphs $A_{n}, D_{n}$, $E_{6}, E_{7}$, or $E_{8}$ and $\Pi$ is an admissible automorphism group of $\mathbf{Z} \Delta$ [4].

We will concentrate on the problem of describing the stable Auslander-algebras by quivers and relations. First we recall the definition: The stable Auslander-algebra $\bar{E}_{\Lambda}$ is the residue algebra of $E_{\Lambda}$ by the two-sided ideal $\sum E_{\Lambda} e_{i} E_{\Lambda}$, where $e_{i}: M \rightarrow M_{i}$ is the canonical projection and where the sum is taken over those $i$ for which $M_{i}$ is not

Received by the editors February 8, 1983

1980 Mathematics Subject Classification. Primary 16A46.

Key words and phrases. Auslander-algebra, Auslander-Reiten quiver.

" 1984 American Mathematical Society $0(0) 2-9947 / 84 \$ 1.00+\$ .25$ per page 
stable. Let us decompose $\bar{E}_{.1}=B_{1} \times B_{2} \times \cdots \times B_{1}$ into a product of connected subalgebras, the so-called stable blocks of $E_{1}$ [5, 1.3]. Since the quiver of $\bar{E}_{1}$ is ${ }_{s} \Gamma_{1}$, the quiver of each $B_{j}$ has the form $\mathbf{Z} \Delta / \Pi$, and we say that $\Delta$ is the class of $B_{j}$. We call a stable block $B$ with quiver $\Gamma$ standard if $B$ is isomorphic to the residue algebra of $k \Gamma$ modulo the ideal of mesh-relations of $\Gamma$ and nonstandard otherwise. Note that an algebra $\Lambda$ whose Auslander-algebra has a nonstandard stable block is necessarily nonstandard itself. It would be interesting to know if the converse of this statement is true. For the known examples of basic and connected nonstandard algebras even more is true: If for two such algebras $\Lambda$ and $\Lambda^{\prime}$ there is an isomorphism between $\bar{E}_{.}$ and $\bar{E}_{\Lambda^{\prime}}$ such that the induced isomorphism from ${ }_{s} \Gamma_{\Lambda}$ to ${ }_{s} \Gamma_{\Lambda^{\prime}}$ extends to an isomorphism from $\Gamma_{\Lambda}$ to $\Gamma_{\Lambda^{\prime}}$, then $\Lambda$ and $\Lambda^{\prime}$ are isomorphic.

With each stable block $\Gamma$ of class $\Delta=A_{n}$ or $D_{n}$, we will associate an integer $N(\Gamma) \geqslant-1$, which for a given $\Delta$ only depends on the group $\Pi$ and is equal to -1 except for a finite number of groups. If $N(\Gamma) \geqslant 0$ we will define algebras $B_{0}$, $B_{1}, \ldots, B_{N(\Gamma)}$ with quiver $\Gamma$ so that the following holds.

THEOREM. If $k$ has characteristic $\neq 2$, every stable block of class $A_{n}$ or $D_{n}$ is standard. If $k$ has characteristic 2 , every stable block $B$ with quiver $\Gamma$ of class $A_{n}$ or $D_{n}$ is standard, unless $N(\Gamma) \geqslant 0$, in which case $B$ is either standard or isomorphic to $B_{0}$, $B_{1}, \ldots$, or $B_{N(\Gamma)}$.

In case $k$ has characteristic 2 and $N(\Gamma) \geqslant 0$, we will also show that $B_{0}$ is not standard and not isomorphic to $B_{1}, \ldots, B_{N(\Gamma)}$. However, for $N(\Gamma) \geqslant 1$, isomorphisms might exist between some of the $B_{1}, \ldots, B_{N\left(\Gamma^{\prime}\right)}$, and some of them might be standard. Computations carried out in the lowest dimensional cases indicate that this does not happen. However, since no $\Gamma$ with $N(\Gamma) \geqslant 1$ has been realized yet as a connected component of any stable part ${ }_{s} \Gamma_{\Lambda}$, it does not seem worthwhile to pursue the matter, in particular since the necessary computations are intricate.

It would be interesting to know which algebras $B$ do arise as stable blocks of Auslander-algebras and what their quivers are. In particular, it is not known whether there is a stable block with quiver $\Gamma$ for which $N(\Gamma) \geqslant 1$ and whether nonstandard stable blocks of class $A_{n}$ or $E_{6}, E_{7}$, or $E_{8}$ exist. The $E_{m}$-cases are not treated here. It is clear that nonstandard stable $E_{m}$-blocks are possible for only finitely many quivers.

I wish to thank the Departments of Mathematics of the University of Basel and the University of Washington for their hospitality and the Schweizerischer Nationalfonds for its support.

2. Results. Throughout the paper we assume $k$ to be algebraically closed. We now fix the notations that are necessary to state our results.

For the vertices of $\mathbf{Z} A_{n}$ and $\mathbf{Z} D_{n}$ we will use the coordinates introduced in [5, 1.3]; i.e., each vertex is represented by a pair $(i, j)$ with $i, j \in \mathbf{Z}$ and $1 \leqslant j \leqslant n$, and $\tau$ is given by $\tau(i, j)=(i-1, j)$. In the case $\mathbf{Z} A_{n}$, there is an arrow ("going up") $(i, j) \rightarrow(i, j+1)$ and an arrow ("going down") $(i, j+1) \rightarrow(i+1, j)$ for each $i \in \mathbf{Z}$ and $1 \leqslant j \leqslant n-1$. In the case $\mathbf{Z} D_{n}$, there are arrows $(i, j) \rightarrow(i, j+1)$ and $(i, j+1) \rightarrow(i+1, j)$ for $i \in \mathbf{Z}$ and $1 \leqslant j \leqslant n-2$ and arrows $(i, n-2) \rightarrow(i, n)$ 
and $(i, n) \rightarrow(i+1, n-2)$ for $i \in \mathbf{Z}$. The fundamental group $\Pi$ is always infinite cyclic, say generated by $\Psi[4,4.2]$.

Consider the case $\Delta=A_{n}$. If $n$ is odd, $\Psi$ can be chosen to be either $\tau^{r}$ or $\tau^{r} \Phi$ for some $r \geqslant 1$, where $\Phi$ is the reflection of $\mathbf{Z} A_{n}$ which fixes all vertices $(i,(n+1) / 2)$ on the "symmetry axis". In coordinates, $\Phi$ is given by

$$
\Phi(i, j)=(i+j-(n+1) / 2, n+1-j) .
$$

In case $n$ is even, we have $\Psi=\tau^{r}$ or $\Psi=\tau^{r} \rho$ for some $r \geqslant 1$, where $\rho$ is the reflection-translation given by

$$
\rho(i, j)=(i+j-1-n / 2, n+1-j) .
$$

Set $\Gamma=\mathbf{Z} A_{n} / \Psi^{\mathbf{Z}}$ with $\Psi$ as above, and let $\pi: \mathbf{Z} A_{n} \rightarrow \Gamma$ be the canonical projection. Define

$$
N(\Gamma)= \begin{cases}{\left[\frac{n-2 r-3}{4 r}\right]} & \text { for } n \text { odd } \\ {\left[\frac{n-2 r-4}{4 r+2}\right]} & \text { for } n \text { even }\end{cases}
$$

where $[x]$ is the greatest integer less than or equal to $x$. By $J$ we denote the ideal of the path algebra $k \Gamma$ generated by the mesh-relations. For each integer $c$ with $0 \leqslant c \leqslant N(\Gamma)$, we set

$$
x_{c}= \begin{cases}(-1,(n+1) / 2+2 c r+r+1) & \text { for } n \text { odd } \\ (-1, n / 2+c(2 r+1)+r+2) & \text { for } n \text { even, }\end{cases}
$$

and we let $v_{c}$ be the only path (going down along the diagonal) in $\mathbf{Z} A_{n}$ from $x_{c}$ to $\Psi^{-(2 c+1)} \tau^{-1} x_{c}$. We define $J_{c}$ to be the ideal of $k \Gamma$ which is generated by all paths of length $n$ together with all mesh-relations, except that we add $\pi\left(v_{c}\right)$ to the mesh-relation arising from the mesh starting at $\pi\left(x_{c}\right)$.

Let $\Delta=D_{n}$, and let $\Psi$ be the generator of an admissible group of automorphisms $\Pi$ of $\mathbf{Z} D_{n}$. If $n \geqslant 5$, we have either $\Psi=\tau^{r}$ or $\Psi=\tau^{r} \Phi$ for some $r \geqslant 1$, where $\Phi$ is the automorphism of $\mathbf{Z} D_{n}$ which exchanges $(i, n-1)$ and $(i, n)$ for all $i \in \mathbf{Z}$ and fixes all the other vertices. For $\mathbf{Z} D_{4}, \Psi$ can be $\tau^{r}, \tau^{r} \Phi$ or $\tau^{r} \rho$ for some $r \geqslant 1$, where $\Phi$ is as above and $\rho$ is given by

$$
\rho(i, j)= \begin{cases}(i, 2) & \text { for } j=2 \\ (i-1,3) & \text { for } j=1 \\ (i, 4) & \text { for } j=3 \\ (i+1,1) & \text { for } j=4\end{cases}
$$

for any $i \in \mathbf{Z}$.

Set $\Gamma=\mathbf{Z} D_{n} / \Psi^{\mathbf{Z}}$ with $\Psi$ as above, and let $\pi: \mathbf{Z} D_{n} \rightarrow \Gamma$ be the canonical projection. Define

$$
N(\Gamma)= \begin{cases}{\left[\frac{n-3-r}{2 r}\right]} & \begin{array}{l}
\text { if either } \Psi=\tau^{r} \text { with } r \text { odd or } \Psi=\tau^{r} \Phi \text { with } r \text { even, } \\
\text { otherwise. }
\end{array}\end{cases}
$$


By $J$ we denote the ideal of the path algebra $k \Gamma$ generated by the mesh-relations. We set $x=(-1, n-1)$, and for each integer $c$ with $0 \leqslant c \leqslant N(\Gamma)$, we let $v_{c}$ be any path in $\mathbf{Z} D_{n}$ from $x$ to $\Psi^{-(2 c+1)} \tau^{-1} x$ for which all vertices $(i, j)$ except the first one and the last one satisfy $j \leqslant n-2$. We define $J_{c}$ to be the ideal of $k \Gamma$ generated by all paths of length $2 n-3$ together with all mesh-relations, except that we add $\pi\left(v_{c}\right)$ to the mesh-relation arising from the mesh starting at $\pi(x)$.

THEOREM. Let $B$ be a stable block with quiver $\Gamma=\mathbf{Z} A_{n} / \Pi$ or $\Gamma=\mathbf{Z} D_{n} / \Pi$. If either $k$ has characteristic $\neq 2$ or $N(\Gamma)=-1$, then $B$ is isomorphic to $k \Gamma / J$ and thus standard. If the characteristic of $k$ equals 2 and $N(\Gamma) \geqslant 0$, then $B$ is isomorphic to either $k \Gamma / J$ or $k \Gamma / J_{c}$ for some $c$ with $0 \leqslant c \leqslant N(\Gamma)$. In this case, $k \Gamma / J_{0}$ is not isomorphic to $k \Gamma / J$ nor to $k \Gamma / J_{c}$ for any $c \geqslant 1$.

3. Proofs. We will give the proof for stable blocks of class $D_{n}$ only. The case of stable $A_{n}$-blocks can be handled with the same methods; it is somewhat simpler since the morphisms in $k\left(\mathbf{Z} A_{n}\right)$ are easier to describe. In addition, the theorem was partially proved in [5] for the $A_{n}$-case.

3.1. Notations (compare [7]). We call a vertex $(i, j)$ of $\mathbf{Z} D_{n}$ low if $j \leqslant n-2$ and high if $j \geqslant n-1$. A high vertex $(i, j)$ is said to be even or odd if $i+j$ is even or odd, respectively. Note that for each integer $i$ there is an even and an odd high vertex with first coordinate $i$. Two high vertices $(i, j)$ and $(p, q)$ will be called congruent if they are either both even or both odd.

For each integer $i$, there are three paths from $(i, n-2)$ to $(i+1, n-2)$ in $\mathbf{Z} D_{n}$ :

$$
\begin{aligned}
& l_{i}:(i, n-2) \rightarrow(i+1, n-3) \rightarrow(i+1, n-2), \\
& h_{i}:(i, n-2) \rightarrow(i, j) \rightarrow(i+1, n-2), \\
& h_{i}^{\prime}:(i, n-2) \rightarrow\left(i, j^{\prime}\right) \rightarrow(i+1, n-2),
\end{aligned}
$$

where $(i, j)$ and $\left(i, j^{\prime}\right)$ are the even and the odd high vertices with first coordinate $i$, respectively. We call $h_{i}$ and $h_{i}^{\prime}$ the even and the odd crenel path. A path $v$ is said to be low if it does not contain a crenel path and high otherwise. Observe that a low path may start or end at a high vertex.

Let $v=\alpha_{r} \alpha_{r-1} \cdots \alpha_{1}$ be a path in $\mathbf{Z} D_{n}$. We denote the image of $v$ in the mesh-category $k\left(\mathbf{Z} D_{n}\right)$ by $\bar{v}=\bar{\alpha}_{r} \cdots \bar{\alpha}_{1}$. We set $\tilde{v}=\tilde{\alpha}_{r} \cdots \tilde{\alpha}_{1}$, where $\tilde{\alpha}=\bar{\alpha}$ for all arrows $\alpha$ whose head or tail is high as well as for $\alpha:(i, j) \rightarrow(i+1, j-1)$ with $2 \leqslant j \leqslant n-2$ and where $\tilde{\alpha}=(-1)^{n-j} \bar{\alpha}$ for all arrows $\alpha:(i, j) \rightarrow(i, j+1)$ with $1 \leqslant j \leqslant n-3$. Sending $v$ to $\tilde{v}$ induces a $k$-linear functor from the path category $k \mathbf{Z} D_{n}$ onto $k\left(\mathbf{Z} D_{n}\right)$; we denote its kernel by $\tilde{I}$ and call it the ideal of modified mesh-relations. Define a path $v$ to be essential if $\tilde{v} \neq 0$.

Two paths $w$ and $w^{\prime}$ from $x$ to $y$ are said to be neighbors if $w=w_{2} v w_{1}$ and $w^{\prime}=w_{2} v^{\prime} w_{1}$ and if the set $\left\{v, v^{\prime}\right\}$ equals $\left\{l_{i+1} h_{i}, h_{i+1} h_{i}\right\}, \quad\left\{h_{i+1} l_{i}, h_{i+1} h_{i}\right\}$, $\left\{l_{i+1} h_{i}^{\prime}, h_{i+1}^{\prime} h_{i}^{\prime}\right\}$, or $\left\{h_{i+1}^{\prime} l_{i}, h_{i+1}^{\prime} h_{i}^{\prime}\right\}$ for some $i \in \mathbf{Z}$ or $\{\beta \alpha, \delta \gamma\}$, where $\alpha, \beta, \gamma, \delta$ are the arrows of Figure 1 for some vertex $(i, j)$ with $2 \leqslant j \leqslant n-3$. 


$$
\begin{array}{ccccc} 
& & (i, j+1) & & \\
(i, j) & & & \searrow \beta & (i+1, j) \\
& \gamma \searrow & \nearrow \delta & \\
& & (i+1, j-1) & &
\end{array}
$$

FIGURE 1

If there is a sequence $w=w_{0}, w_{1}, \ldots, w_{s}=w^{\prime}$ of successive neighbors, $w$ and $w^{\prime}$ are called homotopic. Differences of homotopic paths lie in $\tilde{I}$, since $\beta \alpha-\delta \gamma$ clearly does (Figure 1) and since e.g.

$$
l_{i+1} h_{i}-h_{i+1} h_{i}=\left(l_{i+1}-h_{i+1}-h_{i+1}^{\prime}\right) h_{i}+h_{i+1}^{\prime} h_{i} .
$$

Note that $h_{i+1}^{\prime} h_{i}$ contains a subpath $(i, j) \rightarrow(i+1, n-2) \rightarrow(i+1, j)$ with $j \geqslant$ $n-1$, which is a (modified) mesh-relation.

3.2. Morphisms in $k\left(\mathbf{Z} D_{n}\right)$. We fix two vertices $(i, j)$ and $(p, q)$ of $\mathbf{Z} D_{n}$ such that there exists a path from $(i, j)$ to $(p, q)$. Equivalently, we assume that $i \leqslant p$ and $i+\min (j, n-1) \leqslant p+\min (q, n-1)$, where min denotes the minimum.

In case $p \leqslant i+\min (j, n-1)-1$, we let $u_{0}$ be the path

$$
u_{0}:(i, j) \rightarrow(p, i+\min (j, n-1)-p) \rightarrow(p, q) .
$$

Thus $u_{0}$ is defined if $(p, q)$ lies in the area hatched vertically in Figure 2. In case both $(i, j)$ and $(p, q)$ are low and if in addition $p<i+n-1 \leqslant p+q \leqslant i+j+$ $n-2$, we let $u_{1}$ and $u_{1}^{\prime}$ be the paths

$$
\begin{aligned}
& u_{1}:(i, j) \rightarrow(i, n-2) \stackrel{h_{i}}{\rightarrow}(i+1, n-2) \rightarrow(p, i+n-1-p) \rightarrow(p, q), \\
& u_{1}^{\prime}:(i, j) \rightarrow(i, n-2) \stackrel{h_{1}^{\prime}}{\rightarrow}(i+1, n-2) \rightarrow(p, i+n-1-p) \rightarrow(p, q) .
\end{aligned}
$$

In this case, $(p, q)$ belongs to the area hatched horizontally in Figure 2 .

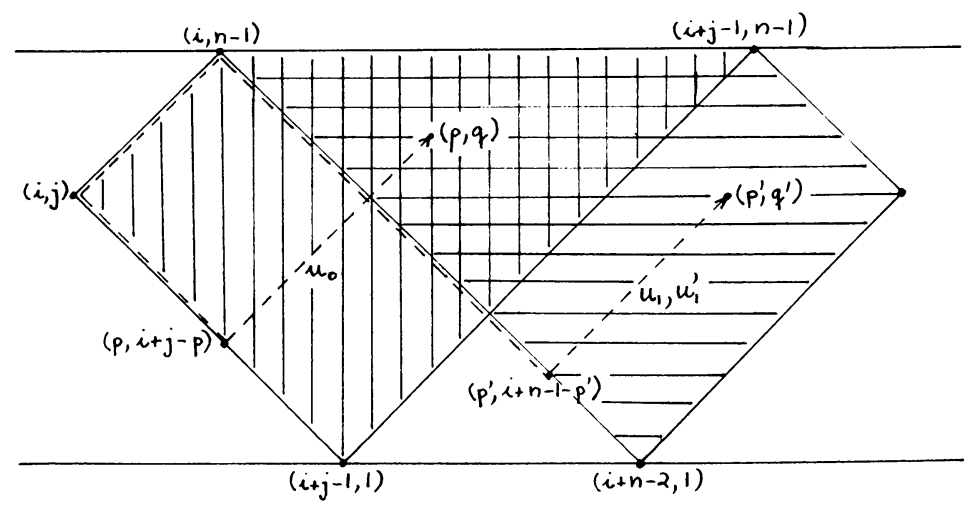

FIGURE 2 
Proposition. Let $(i, j)$ and $(p, q)$ be two vertices of $\mathbf{Z} D_{n}$ such that there exists a path from $(i, j)$ to $(p, q)$. Then

$$
k\left(\mathbf{Z} D_{n}\right)((i, j),(p, q))=\left\{\begin{array}{c}
k \tilde{u}_{0} \quad \text { if }(i, j),(p, q) \text { are low and } p \leqslant i+j-1, \\
p+q \leqslant i+n-2, \\
k \tilde{u}_{0} \quad \text { if }(i, j) \text { is low, }(p, q) \text { is high and } p \leqslant i+j-1, \\
k \tilde{u}_{0} \quad \text { if }(i, j) \text { is high, }(p, q) \text { is low and } p \leqslant i+n-2, \\
k \tilde{u}_{0} \quad \text { if }(i, j),(p, q) \text { are high and congruent and } \\
p \leqslant i+n-2, \\
k \tilde{u}_{0} \oplus k \tilde{u}_{1} \oplus k \tilde{u}_{1}^{\prime} / k\left(-\tilde{u}_{0}+\tilde{u}_{1}+\tilde{u}_{1}^{\prime}\right) \\
\text { if }(i, j),(p, q) \text { are low and } i+n-1 \leqslant p+q, \\
p \leqslant i+j-1, \\
k \tilde{u}_{1} \oplus k \tilde{u}_{1}^{\prime} / k\left(\tilde{u}_{1}+\tilde{u}_{1}^{\prime}\right) \\
\text { if }(i, j),(p, q) \text { are low and } i+j \leqslant p \leqslant \\
i+n-2<p+q \leqslant i+j+n-2, \\
\text { otherwise. }
\end{array}\right.
$$

The proof is based on the following

LEMma. Let $v:(i, j) \rightarrow(p, q)$ be essential.

(a) Assume both $(i, j)$ and $(p, q)$ are low. If $v$ is low, then

$$
p \leqslant i+j-1
$$

and $v$ is homotopic to $u_{0}$. If $v$ is high, then

$$
i+n-1 \leqslant p+q \leqslant i+j+n-2 \text { and } p \leqslant i+n-2 \text {, }
$$

and $v$ is homotopic to $u_{1}$ or $u_{1}^{\prime}$.

(b) If at least one of the vertices $(i, j)$ and $(p, q)$ is high, then

$$
p \leqslant i+\min (j-1, n-2) \text { and } \tilde{v}=\tilde{u}_{0} .
$$

Proof. (a) Assume $v$ is low and suppose $i+j \leqslant p$. Then $v$ is homotopic to a path containing the subpath

$$
(i, j) \rightarrow(i+j-1,1) \rightarrow(i+j-1,2) \rightarrow(i+j, 1),
$$

which is not essential. Thus $p \leqslant i+j-1$, and $v$ is homotopic to $u_{0}$. Observe that any two low paths are homotopic.

If $v$ is high, it has the form $v=v_{2} v_{1} v_{0}$, where $v_{0}:(i, j) \rightarrow(k, n-2)$ is low and $v_{1}=h_{k}$ or $v_{1}=h_{k}^{\prime}$. Since there exists a path $v_{2}$ from $(k+1, n-2)$ to $(p, q)$, we have $i+n-1 \leqslant k+n-1 \leqslant p+q$. Now suppose $v$ contains more than one crenel path. Then we find a subpath $w=w_{2} w_{1} w_{0}$ in $v$ such that $w_{1}$ is low and $w_{0}$ and $w_{2}$ belong to $\left\{h_{k_{1}}, h_{k_{1}}^{\prime}\right\}$ and $\left\{h_{k_{2}}, h_{k_{2}}^{\prime}\right\}$, respectively, for some $k_{1}<k_{2}$. Up to homotopy, we may assume $w_{1}=l_{k_{2}-1} l_{k_{2}-2} \cdots l_{k_{1}+1}$. Since $l_{k_{1}+1} h_{k_{1}}$ is homotopic to $h_{k_{1}+1} l_{k_{1}}$ and $l_{k_{1}+1} h_{k_{1}}^{\prime}$ to $h_{k_{1}+1}^{\prime} l_{k_{1}}$, we reduce to $k_{2}=k_{1}+1$, that is, to the case where $w_{1}$ is the constant path at $\left(k_{1}+1, n-2\right)$. But neither $h_{k_{1}+1} h_{k_{1}}^{\prime}$ nor $h_{k_{1}+1}^{\prime} h_{k_{1}}$ is essential, so that we obtain either $w=h_{k_{1}+1} h_{k_{1}}$, which is homotopic to $l_{k_{1}+1} h_{k_{1}}$, or $w=h_{k_{1}+1}^{\prime} h_{k_{1}}^{\prime}$, which is homotopic to $l_{k_{1}+1} h_{k_{1}}^{\prime}$. Repeating the same argument we 
eventually reach a path $v^{\prime}$ homotopic to $v$ containing just one crenel path, and by "pushing the crenel to the left" as above, we may assume that either $v^{\prime}=v_{1} h_{i} v_{0}$ or $v^{\prime}=v_{1} h_{i}^{\prime} v_{0}$ with low paths $v_{0}:(i, j) \rightarrow(i, n-2)$ and $v_{1}:(i+1, n-2) \rightarrow(p, q)$. Since $v_{1}$ is essential, we get that $p \leqslant i+n-2$. Changing $v_{1}$ modulo homotopy, we obtain $u_{1}$ or $u_{1}^{\prime}$. If we push the crenel to the right as far as possible, we reach $w_{1} h_{p+q+1-n} w_{0}$ or $w_{1} h_{p+q+1-n}^{\prime} w_{0}$, and since $w_{0}:(i, j) \rightarrow(p+q+1-n, n-2)$ is low and essential, we must have $p+q+1-n \leqslant i+j-1$.

(b) Let $w$ be the subpath of $v$ obtained by deleting the first arrow of $v$ is $(i, j)$ is high and the last arrow if $(p, q)$ is high. We are done if $w$ is low. Otherwise, we may assume that $w=w^{\prime} h_{i+1}$ or $w=w^{\prime} h_{i+1}^{\prime}$ for some low $w^{\prime}$ in case $(i, j)$ is high. If $(i, j)$ is even, we have $\tilde{h}_{i+1} \tilde{\alpha}=\tilde{l}_{i+1} \tilde{\alpha}$ and $\tilde{h}_{i+1}^{\prime} \tilde{\alpha}=0$, where $\alpha:(i, j) \rightarrow(i+1, n-2)$ is the first arrow of $v$. If $(i, j)$ is odd, then $\tilde{h}_{i+1} \tilde{\alpha}=0$ and $\tilde{h}_{i+1}^{\prime} \tilde{\alpha}=\tilde{l}_{i+1} \tilde{\alpha}$. In case $(i, j)$ is low and $(p, q)$ is high, we use the same argument for the decomposition $w=w^{\prime} h_{p-1}$ or $w=w^{\prime} h_{p-1}^{\prime}$ for some low $w^{\prime}$ (see end of (a)). In any case, we may replace $w$ by a low path without changing $\tilde{v}$. Therefore, we must have

$$
p \leqslant i+\min (j-1, n-2) .
$$

Proof of the proposition. The lemma implies that the subsets of $\left\{\tilde{u}_{0}, \tilde{u}_{1}, \tilde{u}_{1}^{\prime}\right\}$ listed in the proposition span $k\left(\mathbf{Z} D_{n}\right)((i, j),(p, q))$. If both $(i, j)$ and $(p, q)$ are low and if $p<i+n-1 \leqslant p+q$, we let $u_{0}^{\prime}$ be the low path

$$
u_{0}^{\prime}:(i, j) \rightarrow(i, n-2) \stackrel{l_{i}}{\rightarrow}(i+1, n-2) \rightarrow(p, i+n-1-p) \rightarrow(p, q) .
$$

Then $u_{0}^{\prime}$ is essential if and only if $p \leqslant i+j-1$, in which case it is homotopic to $u_{0}$. Since $-\tilde{l}_{1}+\tilde{h}_{i}+\tilde{h}_{i}^{\prime}=0$, we obtain the following relations:

$$
\begin{array}{ll}
-\tilde{u}_{0}+\tilde{u}_{1}+\tilde{u}_{1}^{\prime}=0 & \text { if } p \leqslant i+j-1, \\
\tilde{u}_{1}+\tilde{u}_{1}^{\prime}=0 & \text { if } i+j \leqslant p .
\end{array}
$$

To finish the proof, it suffices to show that $k\left(\mathbf{Z} D_{n}\right)((i, j),(p, q))$ has the dimension claimed in the proposition. This follows from $[3,6.5]$ by computing the additive function on $\mathbf{Z} D_{n}$ starting at $(i, j)$ (compare [6, 2]). Notice that $k\left(\mathbf{Z} D_{n}\right)((i, j),(p, q))$ $=0$ if $(i, j)$ and $(p, q)$ are both high but not congruent.

3.3. Idea of the proof of the theorem. Let $\Gamma=\mathbf{Z} D_{n} / \Psi^{\mathbf{z}}$, where $\Psi$ is one of the generators described in $\S 2$, and let $\pi: \mathbf{Z} D_{n} \rightarrow \Gamma$ be the canonical projection. Choose a representation-finite algebra $\Lambda$ such that $\Gamma$ arises in the decomposition ${ }_{s} \Gamma_{\Lambda}=\Gamma_{1} \dot{U}$ $\cdots \dot{\cup} \Gamma_{t}$ of the stable part of $\Gamma_{\Lambda}$ into connected components, say $\Gamma=\Gamma_{1}$ (see Introduction). By ind $\Lambda$ we denote the full subcategory of mod $\Lambda$ whose objects are specific representatives of the indecomposables, where $\bmod \Lambda$ is the category of all finite-dimensional $\Lambda$-modules. We let ind $\Lambda$ be the residue category of ind $\Lambda$ modulo the ideal of morphisms which factor through a direct sum of transjective indecomposables. Recall that $X$ is transjective (or equivalently nonstable) if $\tau^{r} X$ is projective for some $r \geqslant 0$, where $\tau=D T r$ is the Auslander-Reiten translation of ind $\Lambda$. We obtain a decomposition

$$
\text { ind } \Lambda=\mathscr{B}_{1} \times \mathscr{B}_{2} \times \cdots \times \mathscr{R}_{t} \text {, }
$$


where each "block" $\mathscr{G}_{j}$ is the residue category of the path category $k \Gamma_{j}$ modulo some ideal $I_{j}$. We will prove that for $\mathscr{G}=\mathscr{T}_{1}, I_{1}$ can be chosen to be among the ideals described in the theorem, viewed as ideals in the path category $k \Gamma$. This is sufficient, since the stable block $B$ of the Auslander-algebra $E_{.1}$ belonging to $\Gamma$ is given by the same quiver and relations as $\mathscr{B}$; in fact,

$$
B=\bigoplus_{x, y \in \cup h} \mathscr{B}(x, y) .
$$

By [4], there exists a well-behaved functor $F: k\left(\mathbf{Z}_{n}\right) \rightarrow$ ind $\Lambda$ (with image 9 ); i.e., a $k$-linear functor with the properties that $F x=\pi x$ for all vertices and that $F \bar{\alpha}$ is irreducible for all arrows of $\mathbf{Z} D_{n}$. For any two vertices $x$ and $y$ of $\mathbf{Z} D_{n}, F$ induces isomorphisms

$$
\begin{aligned}
& \bigoplus_{\pi z=\pi y} k\left(\mathbf{Z} D_{n}\right)(x, z) \rightarrow \text { ind } \Lambda(\pi x, \pi y), \\
& \bigoplus_{\pi z=\pi x} k\left(\mathbf{Z} D_{n}\right)(z, y) \rightarrow \text { ind } \Lambda(\pi x, \pi y) .
\end{aligned}
$$

In other words, $F$ is a covering functor [2].

Our aim is to construct a well-behaved functor $F_{m}: k\left(\mathbf{Z} D_{n}\right) \rightarrow$ ind $\Lambda$ for each $m \geqslant 0$ with the property that

$$
F_{m}(\overline{\psi \alpha}) \equiv F_{m} \bar{\alpha} \quad \text { modulo } \Re^{4 m r+1}(\pi x, \pi y)
$$

for all arrows $\alpha: x \rightarrow y$, where $R$ denotes the radical of the category ind $\Lambda$. Recall that $r$ is the natural number associated with the generator $\Psi$ of $\Pi$. If we succeed in finding such an $F_{m}$ for some $m$ with $2 m r \geqslant n-2$, then $F_{m}$ is $\Pi$-invariant, since $\Re^{2 n-3}=0$ by 3.2. The covering functor $H: k(\Gamma) \rightarrow$ ind $\Lambda$ induced by $F_{m}$ is bijective on the objects, and therefore must be an isomorphism. Hence 9 is standard.

If for some $m$ we cannot find $F_{m}$, we will analyze the obstruction and show that $k$ has characteristic 2 , that $N(\Gamma) \geqslant 0$, and that $\mathscr{B}$ is isomorphic to one of the categories $k \Gamma / J_{c}$ for $0 \leqslant c \leqslant N(\Gamma)$.

In the subsections $3.4,3.6$, and 3.7, we construct $F_{m+1}$ from $F_{m}$ if that is possible, and if not, we show what the obstructions are.

3.4. First step. We postpone the case $\Psi=\tau^{r} \rho \in$ Aut $\mathbf{Z} D_{4}$ until 3.5 and assume for now that $\Psi=\tau^{r}$ or $\Psi=\tau^{r} \Phi$.

For $F_{0}$, we choose any well-behaved functor $k\left(\mathbf{Z} D_{n}\right) \rightarrow$ ind $\Lambda$. We set $G \delta=F_{0} \tilde{\delta}$ for all arrows $\delta$ whose head and tail lie on or between the "diagonals" $\Psi D_{0}=$ $\{\Psi(0, j): 1 \leqslant j \leqslant n\}$ and $D_{-1}=\{(-1, j): 1 \leqslant j \leqslant n\}$ as well as for $\delta=\beta_{2}$, and we choose

$$
G \gamma_{j}=F_{0}\left(\left(\Psi \gamma_{j}\right)^{\sim}\right)
$$

for $2 \leqslant j \leqslant n$ (see Figure 3 ). 


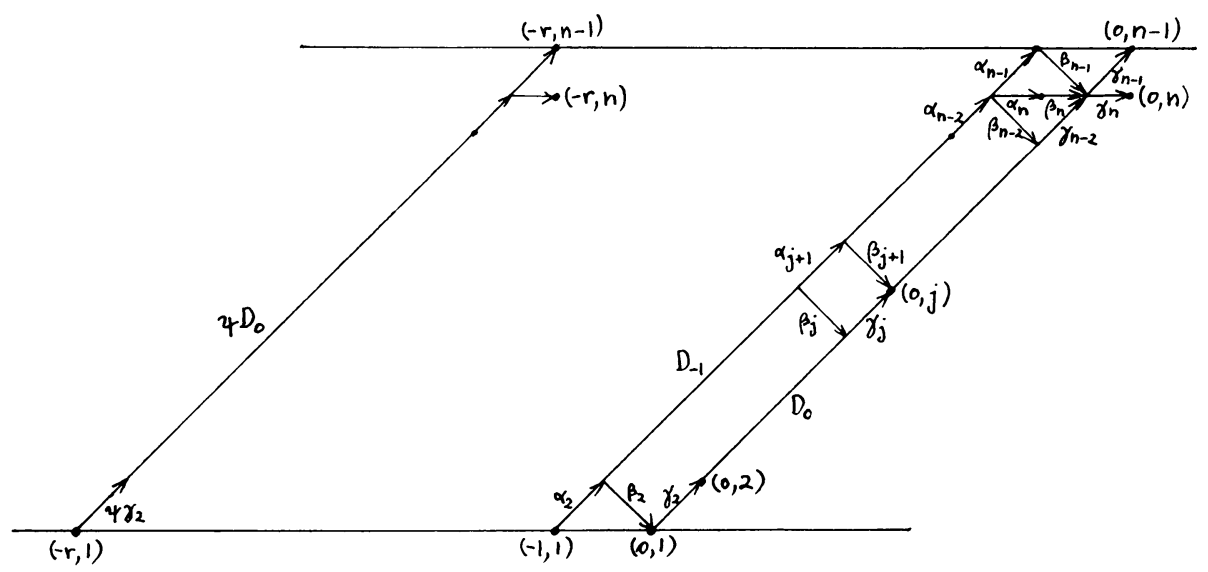

FigURE 3

By induction, we find irreducible morphisms $G \beta_{3}, \ldots, G \beta_{n-1}, G \beta_{n}$ such that

$$
G \beta_{j+1} G \alpha_{j+1}-G \gamma_{j} G \beta_{j}=0 \text { for } j=2, \ldots, n-3,
$$

and

$$
G \beta_{n} G \alpha_{n}+G \beta_{n-1} G \alpha_{n-1}-G \gamma_{n-2} G \beta_{n-2}=0
$$

(compare [5, 1.6 and 7, 3.1]).

Assume $G \beta_{3}, \ldots, G \beta_{j}$ have already been found for some $2 \leqslant j \leqslant n-3$. According to $[4,3.4]$, there exists an Auslander-Reiten sequence

$$
\pi(-1, j) \stackrel{\left[G \alpha_{j+1} G \beta_{j}\right]^{T}}{\rightarrow} \pi(-1, j+1) \oplus \pi(0, j-1) \stackrel{[\beta \gamma]}{\rightarrow} \pi(0, j)
$$

in ind $\Lambda$. Both $\underline{\gamma}$ and $G \gamma_{j}$ are irreducible, and hence

$$
\lambda \underline{\gamma}-G \gamma_{j} \in \Re^{2}(\pi(0, j-1), \pi(0, j))
$$

for some $\lambda \in k \backslash\{0\}$. As $F_{0}$ is a covering functor, we have

$$
\left(\lambda \underline{\gamma}-G \gamma_{j}\right) G \beta_{j}=\sum \lambda_{v} F_{0} \tilde{v}
$$

where $\lambda_{v}$ lies in $k$ and where $v$ is an essential path $v:(-1, j) \rightarrow(s r, j)$ with $s>0$. If $v$ is low, Lemma 3.2 implies that $s r \leqslant j-2$, and $v$ is homotopic to $w \alpha_{j+1}$, where $w$ is the path

$$
w:(-1, j+1) \rightarrow(s r, j-r) \rightarrow(s r, j) .
$$

By 3.2, any high essential path $v$ is of the form $w \alpha_{j+1}$, too, up to homotopy. Thus we may assume that

$$
\left(\lambda \underline{\gamma}-G \gamma_{j}\right) G \beta_{j}=\sum \lambda_{w} F_{0} \tilde{w} F_{0} \tilde{\alpha}_{j+1} .
$$

Set $G \beta_{j+1}=-\lambda \underline{\beta}-\sum \lambda_{w} F_{0} \tilde{w}$. By construction, $G \beta_{j+1}$ satisfies $G \beta_{j+1} G \alpha_{j+1}-$ $G \gamma_{j} G \beta_{j}=0$, since $G \alpha_{j+1}=F_{0} \tilde{\alpha}_{j+1}$. 
In order to define $G \beta_{n-1}$ and $G \beta_{n}$, we start from an Auslander-Reiten sequence

$$
\begin{gathered}
\pi(-1, n-2) \stackrel{\left[G \alpha_{n-1} G \alpha_{n} G \beta_{n-2}\right]^{T}}{\rightarrow} \pi(-1, n-1) \oplus \pi(-1, n) \oplus \pi(0, n-3) \\
\stackrel{\left[\beta \beta^{\prime} \gamma\right]}{\rightarrow} \pi(0, n-2)
\end{gathered}
$$

and use the same argument as above. Observe that any low essential path $v$ : $(-1, n-2) \rightarrow(s r, n-2)$ with $s>0$ is homotopic to $w \gamma_{n-2} \beta_{n-2}=w l_{-1}$ for some $w$, and, since $\tilde{l}_{-1}=\tilde{h}_{-1}+\tilde{h}_{-1}^{\prime}$, any morphism in $\mathrm{GR}^{3}(\pi(-1, n-2), \pi(0, n-2))$ can be written as

$$
\sum \lambda_{w} F_{0} \tilde{w} F_{0} \tilde{\alpha}_{n-1}+\sum \lambda_{w^{\prime}} F_{0} \tilde{w}^{\prime} F_{0} \tilde{\alpha}_{n}
$$

with $\lambda_{w}, \lambda_{w^{\prime}} \in k$.

Let $v:(-1, n-1) \rightarrow \Psi^{-s}(0, n-1)$ be an essential path. By 3.2, we must have $s r \leqslant n-3$ and $\Psi^{-s}(0, n-1)$ must be congruent to $(-1, n-1)$. In case $\Psi=\tau^{r}$, both $r$ and $s$ must be odd, since $\Psi^{-s}(0, n-1)=(s r, n-1)$. If $\Psi=\tau^{r} \Phi$, we have

$$
\Psi^{-s}(0, n-1)= \begin{cases}(2 t r, n-1) & \text { for } s=2 t \\ ((2 t+1) r, n) & \text { for } s=2 t+1,\end{cases}
$$

and hence $r$ is even and $s$ is odd. We conclude that

$$
\begin{aligned}
& \text { ind } \Lambda(\pi(-1, n-1), \pi(0, n-1))=0 \text {, } \\
& \text { ind } \Lambda(\pi(-1, n), \pi(0, n))=0
\end{aligned}
$$

if either $r \geqslant n-2$ or else $r \leqslant n-3$ and either $\Psi=\tau^{r}$ with $r$ even or $\Psi=\tau^{r} \Phi$ with $r$ odd. These are precisely the cases for which $N(\Gamma)=-1$ (see $\$ 2$ ). We conclude that for $N(\Gamma)=-1$, we have

$$
G \gamma_{n-1} G \beta_{n-1}=0, \quad G \gamma_{n} G \beta_{n}=0 .
$$

Extending $G$ by $\Pi$-periodicity to the $\Pi$-orbits of the arrows on which it has already been defined and setting $F_{1} \tilde{\delta}=G \delta$ for all arrows $\delta$, we obtain a well-behaved $\Pi$-invariant functor $F_{1}: k\left(\mathbf{Z} D_{n}\right) \rightarrow$ ind $\Lambda$, and hence $\mathscr{B}$ is standard (3.3).

Assume that $N(\Gamma) \geqslant 0$, and suppose $F_{m}: k\left(\mathbf{Z} D_{n}\right) \rightarrow$ ind $\Lambda$ has been constructed for some $m>0$. We exclude $m=0$ only for the construction of $G$. Set $G \delta=F_{m} \tilde{\delta}$ for all arrows $\delta$ whose head and tail lie on or between $\Psi D_{0}$ and $D_{-1}$ as well as for $\delta=\beta_{2}$, and set $G \gamma_{j}=F_{m}\left(\left(\Psi \gamma_{j}\right)\right.$ ) for $j=3, \ldots, n$ (Figure 3). By induction on $j$, we find irreducible morphisms $G \beta_{j}$ for $j=3, \ldots, n$ such that

$$
G \beta_{j} \equiv F_{m} \tilde{\beta}_{j} \quad \text { modulo } \text { o }^{4 m r+1}(\pi(-1, j), \pi(0, j-1))
$$

and such that the $G \beta_{j}$ satisfy the same relations as in the case $m=0$ above. Assume we already found $G \beta_{3}, \ldots, G \beta_{j}$ for some $2 \leqslant j \leqslant n-3$. Then

$$
\begin{aligned}
\chi & =F_{m} \tilde{\beta}_{j+1} G \alpha_{j+1}-G \gamma_{j} G \beta_{j} \\
& \equiv F_{m} \tilde{\beta}_{j+1} F_{m} \tilde{\alpha}_{j+1}-F_{m} \tilde{\gamma}_{j} F_{m} \tilde{\beta}_{j} \equiv 0 \quad \text { modulo } \stackrel{R}{ }^{4 m r+2}(\pi(-1, j), \pi(0, j)),
\end{aligned}
$$

and as before we can write

$$
\chi=\sum \lambda_{n} F_{m} \tilde{w} F_{m} \tilde{\alpha}_{j+1}
$$


where $\lambda_{w} \in k$ and where $w$ is an essential path from $(-1, j+1)$ to $(s r, j)$ with $s \geqslant 2 m$. We set

$$
G \beta_{j+1}=F_{m} \tilde{\beta}_{j+1}-\sum \lambda_{w} F_{m} \tilde{w}
$$

and we have

$$
\begin{gathered}
G \beta_{j+1} G \alpha_{j+1}-G \gamma_{j} G \beta_{j}=0 \\
G \beta_{j+1} \equiv F_{m} \tilde{\beta}_{j+1} \quad \text { modulo } \mathcal{R}^{4 m r+1}(\pi(-1, j+1), \pi(0, j)) .
\end{gathered}
$$

We obtain $G \beta_{n-1}$ and $G \beta_{n}$ simultaneously by the same argument.

There is no essential path in $\mathbf{Z} D_{n}$ from $(-1, n-1)$ to $\Psi^{-2 m}(0, n-1)$ nor from $(-1, n)$ to $\Psi^{-2 m}(0, n)$ (3.2). We conclude that

$$
\begin{aligned}
\Re^{4 m r+2}(\pi(-1, n-1), \pi(0, n-1)) & =\Re^{4 m r+2 r+2}(\pi(-1, n-1), \pi(0, n-1)), \\
\Re^{4 m r+2}(\pi(-1, n), \pi(0, n)) & =\mathfrak{R}^{4 m r+2 r+2}(\pi(-1, n), \pi(0, n)) .
\end{aligned}
$$

Summing up, we have for any $m$, including $m=0$, that

$$
\begin{aligned}
G \gamma_{n-1} G \beta_{n-1} & \in \mathcal{R}^{4 m r+2 r+2}(\pi(-1, n-1), \pi(0, n-1)), \\
G \gamma_{n} G \beta_{n} & \in \mathcal{R}^{4 m r+2 r+2}(\pi(-1, n), \pi(0, n)) .
\end{aligned}
$$

We extend $G$ to a $k$-linear functor $G: k \mathbf{Z} D_{n} \rightarrow$ ind $\Lambda$ by $\Pi$-periodicity. This functor will be our starting point in 3.6.

3.5. The case $\Gamma=\mathbf{Z} D_{4} /\left(\tau^{r} \rho\right)^{\mathbf{Z}}$. Again we start from a well-behaved functor $F_{0}$ : $k\left(\mathbf{Z} D_{4}\right) \rightarrow$ ind $\Lambda$, but our construction has to be modified slightly. We set $G \delta=F_{0} \tilde{\boldsymbol{\delta}}$ for all arrows $\delta$ whose head and tail both lie on or between the sets $\Psi D$ and $\tau D$, where $D=\{(1,1),(0,2),(0,3),(0,4)\}$, as well as for $\delta=\beta_{2}$, and we choose $G \gamma_{j}=$ $F_{m}\left(\left(\Psi \gamma_{j}\right)\right)$ for $j=2,3,4$ (see Figure 4).

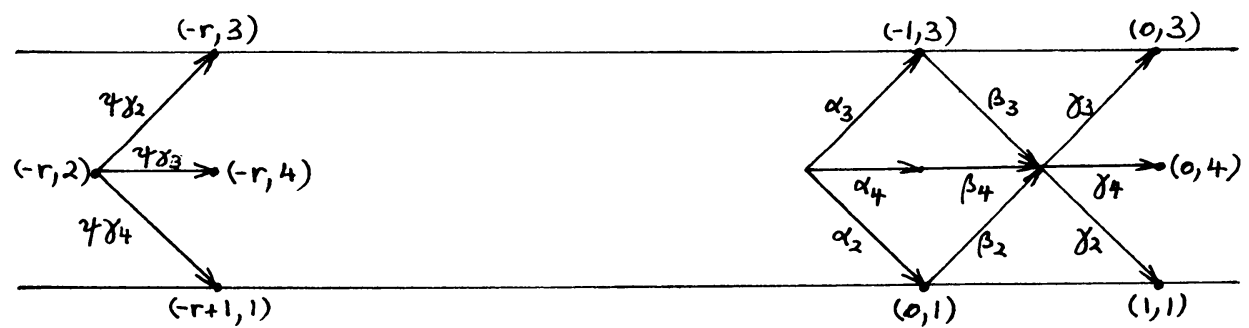

FIGURE 4

There is an Auslander-Reiten sequence

$$
\pi(-1,2) \stackrel{\left[G \alpha_{3} G \alpha_{4} G \alpha_{2}\right]^{T}}{\rightarrow} \pi(-1,3) \oplus \pi(-1,4) \oplus \pi(0,1) \stackrel{\left[\beta_{3} \beta_{4} \beta_{2}\right]}{\rightarrow} \pi(0,2)
$$

in ind $\Lambda$, and we set

$$
G \beta_{j}=\underline{\beta}_{j} \quad \text { for } j=3,4 \text { and } G \beta_{2}=-\underline{\beta}_{2} .
$$

Proposition 3.2 implies that

$$
\text { ind } \Lambda(\pi(-1, j), \pi(0, j))=0
$$


for $j=3,4$, since there is no essential path $v:(-1, j) \rightarrow \Psi^{-s}(0, j)$ for any $s \geqslant 0$. By symmetry,

$$
\text { ind } \Lambda(\pi(0,1), \pi(1,1))=0
$$

holds as well, and thus we see that $G \gamma_{j} G \beta_{j}=0$ for $j=2,3,4$. Extending $G$ by $\Pi$-periodicity and setting $F_{1} \tilde{\delta}=G \delta$ for all arrows yields a well-behaved $\Pi$-invariant functor $F_{1}$. So $\mathscr{G}$ is standard, and the case of quivers $\Gamma$ with $N(\Gamma)=-1$ is settled.

3.6. Second step. From now on, we assume that $N(\Gamma) \geqslant 0$, which means that $r \leqslant n-3$ and that either $\Psi=\tau^{r}$ with $r$ odd or $\Psi=\tau^{r} \Phi$ with $r$ even. By $\vartheta_{x}$ we denote the modified mesh-relation arising from the mesh which starts at the vertex $x$. In 3.4, we obtained a $\Pi$-invariant functor $G: k \mathbf{Z} D_{n} \rightarrow$ ind $\Lambda$ satisfying

$$
\begin{aligned}
& G \vartheta_{x}=0 \text { for } x \notin \Pi(-1, n-1) \cup \Pi(-1, n), \\
& G \vartheta_{(-1, n-1)}, G \vartheta_{(-1, n)} \in \mathbb{R}^{4 m r+2 r+2} .
\end{aligned}
$$

Our aim in this section is to construct a $\Pi$-invariant functor $H: k \mathbf{Z} D_{n} \rightarrow$ ind $\Lambda$ such that

$$
H \vartheta_{x} \in \Re^{4(m+1) r+2}\left(\pi x, \pi \tau^{-1} x\right)
$$

for all $x$, provided that the characteristic of $k$ is different from 2. Of course, $H \alpha$ will still be irreducible for every arrow $\alpha$. We will then find $F_{m+1}$ in 3.7.

Since $\mathcal{R}^{2 n-3}=0$, we may assume that $(2 m+1) r \leqslant n-3$. The two paths

$$
\begin{aligned}
& v:(-1, n-1) \rightarrow((2 m+1) r, n-2-(2 m+1) r) \rightarrow \Psi^{-(2 m+1)}(0, n-1), \\
& v^{\prime}:(-1, n) \rightarrow((2 m+1) r, n-2-(2 m+1) r) \rightarrow \Psi^{-(2 m+1)}(0, n)
\end{aligned}
$$

are both essential, and $\tilde{v}, \tilde{v}^{\prime}$ is a basis of

$$
k\left(\mathbf{Z} D_{n}\right)\left((-1, n-1), \Psi^{-(2 m+1)}(0, n-1)\right), \quad k\left(\mathbf{Z} D_{n}\right),\left((-1, n), \Psi^{-(2 m+1)}(0, n)\right),
$$

respectively, by 3.2 . Since $F_{m}$ is a covering functor, we have

(*) $\quad G \vartheta_{(-1, n-1)} \equiv \lambda^{\prime} F_{m} \tilde{v} \equiv \lambda G v \quad$ modulo $\Re^{4 m r+2 r+3}(\pi(-1, n-1), \pi(0, n-1))$

$$
=\Re^{4(m+1) r+2}(\pi(-1, n-1), \pi(0, n-1))
$$

for some $\lambda, \lambda^{\prime} \in k$, and similarly

$(* *) \quad G \vartheta_{(-1, n)} \equiv \mu G v^{\prime} \quad$ modulo $\Re^{4(m+1) r+2}(\pi(-1, n), \pi(0, n))$

for $\mu \in k$. Observe that the length of a path from $(-1, j)$ to $\Psi^{-s}(0, j)$ is congruent to 2 modulo $2 r$.

We will now change $G$ so as to obtain $\mu=0$. Let $u_{j}$ be the path

$$
u_{j}:(-1, j) \rightarrow\left\{\begin{array}{r}
((2 m+1) r, j-1-(2 m+1) r) \rightarrow((2 m+1) r, j-1) \\
\text { for } j<n, \\
((2 m+1) r, n-2-(2 m+1) r) \rightarrow((2 m+1) r, n-2) \\
\text { for } j=n,
\end{array}\right.
$$

and $j \geqslant(2 m+1) r+2$. Using the notations of Figure 3, we set

$$
\begin{aligned}
& G^{\prime} \beta_{j}=G \beta_{j} \text { for } j \leqslant(2 m+1) r+1, \\
& G^{\prime} \beta_{j}=G \beta_{j}-\mu G u_{j} \text { for } j \geqslant(2 m+1) r+2,
\end{aligned}
$$


and $G^{\prime} \delta=G \delta$ for all arrows $\delta$ whose head and tail lie on or between $\Psi D_{0}$ and $D_{-1}$, and we extend $G^{\prime}$ by $\Pi$-periodicity.

We have $G^{\prime} \vartheta_{(i, j)}=G \vartheta_{(i, j)}=0$ for all $(i, j)$ with $i \not \equiv-1$ modulo $r$ and for $(i, j)=(-1, j)$ with $j<(2 m+1) r+1$. Since any two low paths from $(-1, j)$ to $((2 m+1) r, j)$ are homotopic, $G^{\prime}$ vanishes on $\vartheta_{(-1, j)}$ for $(2 m+1) r+2 \leqslant j \leqslant n-3$ as well. As for $j=(2 m+1) r+1$, we have

$$
G^{\prime} \vartheta_{(-1, j)}=G \vartheta_{(-1, j)}-(-1)^{n-j} \mu G\left(u_{j+1} \alpha_{j+1}\right)=(-1)^{n-j-1} \mu G\left(u_{j+1} \alpha_{j+1}\right)=0 \text {, }
$$

since $u_{j+1} \alpha_{j+1}$ is homotopic to

$$
\begin{aligned}
(-1, j) & =(-1,(2 m+1) r+1) \rightarrow((2 m+1) r-1,1) \\
& \rightarrow((2 m+1) r-1,2) \rightarrow((2 m+1) r, 1) \rightarrow((2 m+1) r, j),
\end{aligned}
$$

which is not essential. Computing $G^{\prime} \vartheta_{(-1, n-2)}$ we get

$$
\begin{aligned}
& G \vartheta_{(-1, n-2)}-\mu\left[G\left(u_{n-1} \alpha_{n-1}\right)+G\left(u_{n} \alpha_{n}\right)-G\left(\left(\Psi^{-(2 m+1)} \gamma_{n-2}\right) u_{n-2}\right)\right] \\
& \quad=-\mu G u G \vartheta_{(-1, n-2)}=0,
\end{aligned}
$$

where $u:(0, n-2) \rightarrow((2 m+1) r, n-2)$ is any low path. Finally, we have

$$
\begin{aligned}
G^{\prime} \vartheta_{(-1, n-1)} & \equiv(\lambda-\mu) G^{\prime} v \quad \text { modulo } \Re^{4(m+1) r+2}, \\
G^{\prime} \vartheta_{(-1, n)} & \equiv 0 \quad \text { modulo } \Re^{4(m+1) r+2} .
\end{aligned}
$$

Without loss of generality, we can therefore assume that $\mu=0$ in the formula $(* *)$ above. If $\lambda$ in (*) is zero as well, we reached our goal, and $G \vartheta_{x}$ lies in $\Re^{4(m+1) r+2}$ for all $x$. In case $\lambda \neq 0$ in $(*)$, we choose $\lambda^{\prime}$ satisfying $\lambda^{\prime 2(2 m+1) r}=\lambda$, and we set $G^{\prime} \delta=\lambda^{\prime} G \delta$ for all arrows $\delta$. Then

$$
G^{\prime} \vartheta_{(-1, n-1)} \equiv G^{\prime} v \quad \text { modulo } \Re^{4(m+1) r+2}(\pi(-1, n-1), \pi(0, n-1)),
$$

or in other words, we can choose $\lambda=1$ in (*).

Until the end of this section, we assume that $k$ does not have characteristic 2 and that $\lambda=1$ in (*). We need a few notations: For $-r \leqslant i \leqslant-2$, we let $\delta_{i}$ and $\varepsilon_{i}$ be the arrows

$$
\delta_{i}:(i, n-2) \rightarrow(i, j), \quad \varepsilon_{i}:\left(i, j^{\prime}\right) \rightarrow(i+1, n-2),
$$

where $(i, j) \neq\left(i, j^{\prime}\right)$ are high and $(i, j)$ is congruent to $(-1, n-1)$. We let $v_{i}$ be the low path

$$
v_{i}:(i, n-2) \rightarrow(i+(2 m+1) r, n-2-(2 m+1) r) \rightarrow(i+(2 m+1) r, n-2)
$$

for each integer $i$, and we choose $u_{j}:(-1, j) \rightarrow((2 m+1) r, j-1)$ as before for $(2 m+1) r+2 \leqslant j$. In addition, we will use the notations of Figure 3 . Set $\chi=$ $\Psi^{-(2 m+1)}$. We define

$$
\begin{aligned}
& H \beta_{n-1}=G \beta_{n-1}-\frac{1}{2} G\left(v_{0} \beta_{n-1}\right), \\
& H \alpha_{n-1}=G \alpha_{n-1}-\frac{1}{2} G\left(\left(\chi \alpha_{n-1}\right) v_{-1}\right), \\
& H \varepsilon_{i}=G \varepsilon_{i}+\frac{1}{2} G\left(v_{i+1} \varepsilon_{i}\right) \text { for }-r \leqslant i \leqslant 2, \\
& H \delta_{i}=G \delta_{i}-\frac{1}{2} G\left(\left(\chi \delta_{i}\right) v_{i}\right) \text { for }-r \leqslant i \leqslant-2, \\
& H \beta_{j}=G \beta_{j}-\frac{1}{2} G u_{j} \text { for }(2 m+1) r+2 \leqslant j \leqslant n-2 .
\end{aligned}
$$




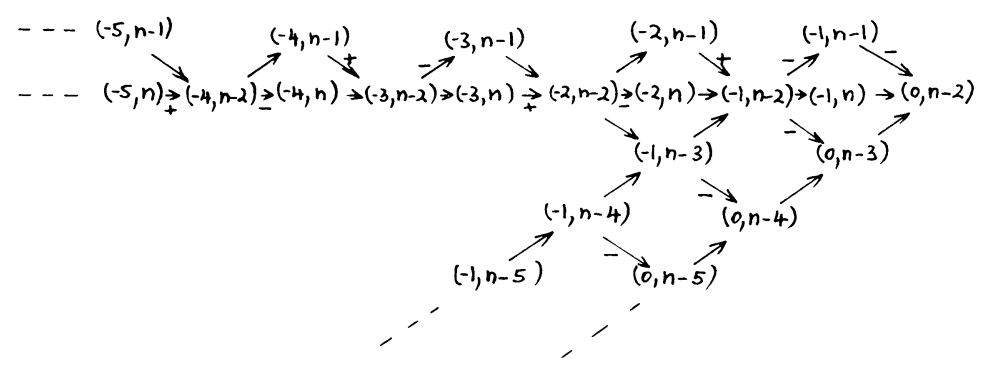

Figure 5

We extend $H$ by $\Pi$-periodicity to the $\Pi$-orbits of the arrows for which $H$ has already been defined, and we let $H$ coincide with $G$ on the remaining arrows. Figure 5 indicates the arrows on which $H$ differs from $G$; they are marked with signs + or according to the definition of $H$ above.

We now check that $H \vartheta_{x} \equiv 0$ for all vertices $x$ of $\mathbf{Z} D_{n}$; all congruences are modulo $\Re^{4(m+1) r+2}$. Since products of "correction terms" in $6 \Re^{2(2 m+1) r+1}$ used to define $H$ lie in $\Re^{4(2 m+1) r+2} \subseteq \Re^{4(m+1) r+2}$, we need not take them into account. In addition, if $w$ and $w^{\prime}$ are homotopic paths of length $2(2 m+1) r+2$, then

$$
G w-G w^{\prime} \in \Re^{2(2 m+1) r+2(2 m+1) r+2},
$$

and hence $G w \equiv G w^{\prime}$.

First notice that $\Psi$ exchanges even and odd high vertices, since $N(\Gamma) \geqslant 0$. In particular, we have that $\Psi(0, n-1)$ is congruent to $(-1, n-1)$, so that

$$
\begin{gathered}
H \gamma_{n-1}=H \delta_{-r}=G \delta_{-r}-\frac{1}{2} G\left(\left(\chi \delta_{-r}\right) v_{-r}\right)=G \gamma_{n-1}-\frac{1}{2} G\left(\left(\chi \gamma_{n-1}\right) v_{0}\right), \\
H \gamma_{n}=G \gamma_{n} .
\end{gathered}
$$

This yields

$$
H \vartheta_{(-1, n-1)}=H\left(\gamma_{n-1} \beta_{n-1}\right) \equiv G \vartheta_{(-1, n-1)}-2 \cdot G\left(\left(\chi \gamma_{n-1}\right) v_{0} \beta_{n-1}\right) \equiv 0
$$

(see $(*)$ with $\lambda=1)$ and

$$
H \vartheta_{(-1, n)}=G \vartheta_{(-1, n)} \equiv 0
$$

(see $(* *)$ with $\mu=0$ ). For any high vertex $(i, j)$ with $-r \leqslant i \leqslant-2$, we either have that $H \vartheta_{(i, j)}=G \vartheta_{(i, j)}=0$ or else $\vartheta_{(i, j)}=\delta_{i+1} \varepsilon_{i}\left(\right.$ with $\left.\delta_{-1}=\alpha_{n-1}\right)$, in which cases

$$
H \vartheta_{(i, j)} \equiv G \vartheta_{(i, j)}=0
$$

because the two contributions in $9 R^{2(2 m+1) r+2}$ cancel.

Clearly, $H \vartheta_{(i, j)} \equiv 0$ for all $(i, j)$ with $j \leqslant n-3$; unless $i \equiv-1$ modulo $r, H$ and $G$ coincide on $\vartheta_{(i, j)}$, and for $i=-1$ the contributions in $\Re^{2(2 m+1) r+2}$ cancel. To check for $(i, n-2)$, we assume that $(-1, n-1)$ is even; if it is odd we just replace any $h_{p}$ by $h_{p}^{\prime}$ and vice versa in what follows. Let $-r \leqslant i \leqslant-2$. Then

$$
\begin{aligned}
H l_{i} & =G l_{i}, \\
H h_{i} & =H\left(\left(\sigma^{-1} \delta_{i}\right) \delta_{i}\right)=G h_{i}-\frac{1}{2} G\left(\left(\chi h_{i}\right) v_{i}\right), \\
H h_{i}^{\prime} & =H\left(\varepsilon_{i}\left(\sigma \varepsilon_{i}\right)\right)=G h_{i}^{\prime}+\frac{1}{2} G\left(v_{i+1}\left(h_{i}^{\prime}\right)\right),
\end{aligned}
$$


where for any arrow $\zeta: x \rightarrow y$ of $\mathbf{Z} D_{n}$ we set $\sigma \zeta: \tau y \rightarrow x$. Observe that $\chi h_{i}=$ $h_{i+(2 m+1) r}^{\prime}$ and that $h_{i+(2 m+1) r}^{\prime} v_{i}$ and $v_{i+1} h_{i}^{\prime}$ are homotopic paths of length $2(2 m+1) r+2$ from (3.2). Adding up, we obtain

$$
H \vartheta_{(i, n-2)}=H h_{i}+H h_{i}^{\prime}-H l_{i} \equiv G \vartheta_{(i, n-2)}=0 .
$$

In the remaining case $i=-1$, we have

$$
\begin{aligned}
H l_{-1} & =H\left(\gamma_{n-2} \beta_{n-2}\right)=G l_{-1}-\frac{1}{2} G\left(\left(\chi \gamma_{n-2}\right) u_{n-2}\right) \\
& \equiv G l_{-1}-\frac{1}{2} G\left(v_{0} l_{-1}\right), \\
H h_{-1} & =H\left(\beta_{n-1} \alpha_{n-1}\right) \equiv G h_{-1}-\frac{1}{2} G\left(\left(\chi \beta_{n-1}\right)\left(\chi \alpha_{n-1}\right) v_{-1}\right)-\frac{1}{2} G\left(v_{0} \beta_{n-1} \alpha_{n-1}\right) \\
& \equiv G h_{-1}-\frac{1}{2} G\left(v_{0} h_{-1}^{\prime}\right)-\frac{1}{2} G\left(v_{0} h_{-1}\right), \\
H h_{-1}^{\prime} & =H\left(\beta_{n} \alpha_{n}\right)=G h_{-1}^{\prime},
\end{aligned}
$$

and adding up yields $H \vartheta_{(-1, n-2)} \equiv 0$.

3.7. Third step.

Proposition. Let $H: k \mathbf{Z} D_{n} \rightarrow$ ind $\Lambda$ be a k-linear functor with $H x=\pi x$ for all vertices and $H \alpha$ irreducible for all arrows of $\mathbf{Z} D_{n}$ and such that for some $t>1$

$$
H \vartheta_{x} \in \Re^{t+1}\left(\pi x, \pi \tau^{-1} x\right)
$$

for all vertices $x$. Then there exists a well-behaved functor $F: k\left(\mathbf{Z} D_{n}\right) \rightarrow$ ind $\Lambda$ with $F \tilde{\alpha} \equiv H \boldsymbol{\alpha}$ modulo $R^{t}$ for all arrows $\boldsymbol{\alpha}$ of $\mathbf{Z} D_{n}$.

If we apply this proposition for $t=4(m+1) r+1$ to the functor $H$ constructed in 3.6, we see that for every arrow $\alpha$

$$
F\left((\Psi \alpha)^{\tilde{}}\right) \equiv H(\Psi \alpha)=H \alpha \equiv F \tilde{\alpha} \quad \operatorname{modulo} \Re^{4(m+1) r+1} .
$$

We can therefore choose $F_{m+1}=F$. We see that we find a well-behaved $F_{m}$ as described in 3.3 for every natural number $m$. As a consequence, $\mathscr{B}$ is standard, provided that the characteristic of $k$ is not 2. Remember that we needed this condition in order to construct $H$ in 3.6.

The proof of the proposition is based on the following

LEMMA. Let $x$ be a vertex of $\mathbf{Z} D_{n}$ and $\alpha_{i}: x \rightarrow y_{i}$ for $i=1, \ldots, s$ the arrows with tail $x$. Given irreducible morphisms $f_{i}: \pi x \rightarrow \pi y_{i}$ and $g_{i}: \pi y_{i} \rightarrow \pi \tau^{-1} x$ in ind $\Lambda$ such that $\sum g_{i} f_{i} \in R^{t+1}\left(\pi x, \pi \tau^{-1} x\right)$ for some $t>1$, there are morphisms $g_{i}^{\prime} \in \operatorname{ind} \Lambda\left(\pi y_{i}, \tau^{-1} x\right)$ with $g_{i}^{\prime}-g_{i} \in \mathcal{R}^{t}\left(\pi y_{i}, \pi \tau^{-1} x\right)$ and such that $\sum g_{i}^{\prime} f_{i}=0$.

Proof. This is the version of $[7,3.7]$ for ind $\Lambda$ of ind $\Lambda$, and we will only indicate the proof here. By $[4,2.2]$ there is a well-behaved functor $G: k\left(\mathbf{Z} D_{n}\right) \rightarrow$ ind $\Lambda$ with $G \bar{\alpha}_{i}=f_{i}$. Therefore

$$
\sum g_{i} f_{i}=\sum \lambda_{w} G \bar{w}
$$

where $w$ ranges over paths from $x$ to $\Psi^{-s} \tau^{-1} x$ with $s \geqslant 0$ whose length is not less than $t+1$. Each $w$ has the form $v \alpha_{i}$ for some $i$ and some $v$ starting at $y_{i}$, so that

$$
\sum_{i} g_{i} f_{i}=\sum_{i}\left(\sum_{v} \lambda_{i, v} G \bar{v}\right) f_{i}
$$


We set

$$
g_{i}^{\prime}=g_{i}-\sum_{v} \lambda_{i, v} G \bar{v}
$$

ProOF OF THE PROPOSITION (COMPARE [7, 3.5]). We go back to the original construction of well-behaved functors in [4, 2.2]. Set $F \tilde{\alpha}=H \alpha$ for all arrows $\alpha$ : $(i, j) \rightarrow(p, q)$ of $\mathbf{Z} D_{n}$ with $i+\min (j, n-1)=0$. Suppose that for some vertex $x$, $F \tilde{\alpha}_{i}$ has been constructed for all arrows $\alpha_{i}: x \rightarrow y_{i}$ with tail $x$. Set

$$
e_{i}= \begin{cases}1 & \text { if }\left(\sigma^{-1} \alpha_{i}\right)^{\tilde{}}\left(\alpha_{i}\right)^{\tilde{}}=\left(\overline{\sigma^{-1} \alpha_{i}}\right) \overline{\alpha_{i}}, \\ -1 & \text { if }\left(\sigma^{-1} \alpha_{i}\right)^{\tilde{}}\left(\alpha_{i}\right)^{\tilde{}}=-\left(\overline{\sigma^{-1} \alpha_{i}}\right) \overline{\alpha_{i}} .\end{cases}
$$

Then

$$
\begin{aligned}
\sum_{i} e_{i} H\left(\sigma^{-1} \alpha_{i}\right) F \tilde{\alpha}_{i} & \equiv \sum_{i} e_{i} H\left(\sigma^{-1} \alpha_{i}\right) H \alpha_{i}=H \vartheta_{x} \\
& \equiv 0 \text { modulo } G^{t+1}\left(\pi x, \pi \tau^{-1} x\right) .
\end{aligned}
$$

We apply the lemma for $f_{i}=e_{i} F\left(\alpha_{i}\right)$ and $g_{i}=H\left(\sigma^{-1} \alpha_{i}\right)$, and we set $F\left(\left(\sigma^{-1} \alpha_{i}\right)\right)=g_{i}^{\prime}$. Clearly, $F \tilde{\vartheta}_{x}=0$. We use the dual arguments if $F$ is already defined on the "right half" of a mesh.

3.8. The case $N(\Gamma) \geqslant 0$ and $k$ of characteristic 2 . From now on, we assume that $k$ has characteristic 2 and that $N(\Gamma) \geqslant 0$; we have shown that $9 B$ is standard in all other cases. Assume we met no obstruction in constructing $F_{0}, F_{1}, \ldots, F_{c}$ for some $c \geqslant 0$ (3.3), but that the functor $G$ we obtained from $F_{c}$ (3.6) satisfies

$$
\begin{aligned}
& G \vartheta_{x}=0 \text { for } x \notin \Pi(-1, n-1) \cup \Pi(-1, n), \\
& G \vartheta_{(-1, n-1)} \equiv G v \text { modulo } \Re^{4(c+1) r+2}(\pi(-1, n-1), \pi(0, n-1)), \\
& G \vartheta_{(-1, n)} \equiv 0 \text { modulo } R_{R}^{4(c+1) r+2}(\pi(-1, n), \pi(0, n)),
\end{aligned}
$$

where $v$ is the path

$$
v:(-1, n-1) \rightarrow((2 c+1) r, n-2-(2 c+1) r) \rightarrow \Psi^{-(2 c+1) r}(0, n-1) .
$$

Notice that $(2 c+1) r \leqslant n-3$ and hence $c \leqslant N(\Gamma)=[(n-3-r) / 2 r]$.

In this section, we will construct a $k$-linear $\Pi$-invariant functor $F_{m}: k \mathbf{Z} D_{n} \rightarrow$ ind $\Lambda$, for all $m \geqslant c+1$, with $F_{m} x=\pi x$ for all vertices and $F_{m} \alpha$ irreducible for all arrows of $\mathbf{Z} D_{n}$ and such that

$$
\begin{aligned}
& F_{m} \vartheta_{x}=0 \text { for } x \notin \Pi(-1, n-1) \cup \Pi(-1, n), \\
& \left.\begin{array}{l}
F_{m} \vartheta_{(-1, n-1)}+F_{m} v \equiv 0 \\
F_{m} \vartheta_{(-1, n)} \equiv 0
\end{array}\right\} \text { modulo } \Re^{4 m r+2} .
\end{aligned}
$$

If we succeed in doing so for some $m$ with $4 m r+2 \geqslant 2 n-3$, then $F_{m}$ induces a $k$-linear functor from $k \Gamma$ to ind $\Lambda$ which vanishes on the ideal $J_{c}$ defined in $\$ 2$. Notice that mesh-relations and modified mesh-relations are the same since $k$ has characteristic 2 . Hence we obtain a functor

$$
H: k \Gamma / J_{c} \rightarrow \text { ind } \Lambda,
$$


and $H$ is bijective on the objects and full since $F_{m}$ is full. Indeed, let $F: k\left(\mathbf{Z} D_{n}\right) \rightarrow$ ind $\Lambda$ be any well-behaved functor. Then $F \tilde{\alpha}$ and $F_{m} \alpha$ differ only by a nonzero scalar modulo $\mathscr{R}^{2}$. By induction, $F \tilde{w}$ and $F_{m} w$ differ by a nonzero scalar modulo $\Re^{t+1}$ for any path $w$ of length $t$. Since $F$ is a covering functor and $R$ is nilpotent, this shows that $F_{m}$ is full.

To see that $H$ is faithful, we count dimensions. First notice that the associated graded category [2], of $k \Gamma / J_{c}$ is the mesh-category $k(\Gamma)$. In fact, the radical of $k \Gamma / J_{c}$ is generated by the residue classes of the arrows of $\Gamma$. Therefore, we have for any pair $x, y$ of vertices of $\mathbf{Z} D_{n}$ :

$$
\begin{aligned}
\operatorname{dim}_{k} k \Gamma / J_{c}(\pi x, \pi y) & =\operatorname{dim}_{k} k(\Gamma)(\pi x, \pi y) \\
& =\sum_{\pi z=\pi y} \operatorname{dim}_{k} k\left(\mathbf{Z} D_{n}\right)(x, z)=\operatorname{dim}_{k} \text { ind } \Lambda(\pi x, \pi y) .
\end{aligned}
$$

Here we use that covering functors exist from $k\left(\mathbf{Z} D_{n}\right)$ to both ind $\Lambda$ and $k(\Gamma)$. Thus $H$ is an isomorphism from $\mathscr{B}_{c}=k \Gamma / J_{c}$ to $\mathscr{G}$.

We choose $F_{c+1}=G$. Assume $F_{m}$ has been found for some $m \geqslant c+1$ such that $4 m r+2 \leqslant 2 n-4$ still holds. For $j=n-1$ or $j=n$ and for each integer $t$ such that $t r \leqslant n-3$, we let $v_{j, t}$ be the path

$$
v_{j, t}:(-1, j) \rightarrow(t r, n-2-t r) \rightarrow \Psi^{-t}(0, j) .
$$

Notice that the path $v:(-1, n-1) \rightarrow \Psi^{-(2 c+1)}(0, n-1)$ above is just $v_{n-1,2 c+1}$. By assumption, we have

$$
\begin{aligned}
& F_{m} \vartheta_{x}=0 \quad \text { for } x \notin \Pi(-1, n-1) \cup \Pi(-1, n), \\
& \left.\begin{array}{l}
F_{m} \vartheta_{(-1, n-1)}+F_{m} v \equiv \lambda F_{m} v_{n-1,2 m+1} \\
F_{m} \vartheta_{(-1, n)} \equiv \mu F_{m} v_{n, 2 m+1}
\end{array}\right\} \text { modulo } \Re^{4(m+1) r+2} .
\end{aligned}
$$

for some $\lambda, \mu \in k$. Remember that

$$
\Re^{4 m r+2}(\pi(-1, j), \pi(0, j))=\Re^{4 m r+2 r+2}(\pi(-1, j), \pi(0, j))
$$

for $j=n-1$ and $j=n$, since there are no essential paths $(-1, j) \rightarrow \Psi^{-2 m}(0, j)$. If $2(2 m+1) r+2 \leqslant 2 n-4$, we have to find an $F_{m+1}$ satisfying the same relations, but with $\lambda=\mu=0$. To define $F_{m+1}$ we use again the notations of Figure 3. For $j=2, \ldots, n$, we let

$$
\begin{aligned}
& u_{j}:(-1, j) \rightarrow(2(m-c) r, \min (n-1, j)-1), \\
& u_{j}^{\prime}:(-1, j) \rightarrow((2 m+1) r, \min (n-1, j)-1),
\end{aligned}
$$

be any low paths (of length $4(m-c) r+2$ and $2(2 m+1) r+2$, respectively).

If $m-c$ is odd, we set

$$
F_{m+1} \beta_{j}=F_{m} \beta_{j}+(\lambda+\mu) F_{m} u_{j}+\lambda F_{m} u_{j}^{\prime},
$$

if $m-c$ is even, we set

$$
F_{m+1} \beta_{j}=F_{m} \beta_{j}+(\lambda+\mu) F_{m} u_{j}+\mu F_{m} u_{j}^{\prime},
$$

for $j=2, \ldots, n$. We extend $F_{m+1}$ to the $\Pi$-orbits of the $\beta_{j}$ by $\Pi$-periodicity, and we let $F_{m+1}$ coincide with $F_{m}$ on all other arrows. 
In order to check that $F_{m+1}$ has the desired properties, we need the following lemma, which we will prove later.

Lemma. For any $s \geqslant 0$ with $(2 s+2 c+1) r \leqslant n-3$ we have that modulo $\Re^{4(s+c+1) r+2}$ :

$$
\begin{aligned}
F_{m} v_{n-1.2 s} & \equiv \begin{cases}0 & \text { if } s \text { is odd }, \\
F_{m} v_{n-1,2 s+2 c+1} & \text { if } \text { s is even },\end{cases} \\
F_{m} v_{n, 2 s} & \equiv \begin{cases}F_{m} v_{n, 2 s+2 c+1} & \text { if } s \text { is odd }, \\
0 & \text { if } s \text { is even } .\end{cases}
\end{aligned}
$$

By definition, we have

$$
F_{m+1} \vartheta_{(i, j)}=F_{m} \vartheta_{(i, j)}=0
$$

for all $(i, j)$ with $i \not \equiv-1$ modulo $r$. As $F_{m}$ vanishes on all low mesh-relations, it is easy to see that $F_{m+1} \vartheta_{(-1, j)}=0$ for $j \leqslant n-3$. As for $(-1, n-2),(-1, n-1)$, and $(-1, n)$, we concentrate on the case that $m-c$ is odd; the other one can be handled in the same way. We obtain

$$
\begin{aligned}
F_{m+1} \vartheta_{(-1, n-2)}= & F_{m} \vartheta_{(-1, n-2)}+(\lambda+\mu)\left(F_{m} u\right) F_{m} \vartheta_{(-1, n-2)} \\
& +\lambda\left(F_{m} u^{\prime}\right) F_{m} \vartheta_{(-1, n-2)}=0,
\end{aligned}
$$

where $u$ and $u^{\prime}$ are low paths from $(0, n-2)$ to $(2(m-c) r, n-2)$ and $((2 m+1) r, n-2)$, respectively. The reason is that we have

$$
\begin{aligned}
& \left(F_{m} \gamma_{n-2}\right) F_{m} u_{n-2}=\left(F_{m} u\right) F_{m}\left(\gamma_{n-2} \beta_{n-2}\right), \\
& \left(F_{m} \gamma_{n-2}\right) F_{m} u_{n-2}^{\prime}=\left(F_{m} u^{\prime}\right) F_{m}\left(\gamma_{n-2} \beta_{n-2}\right),
\end{aligned}
$$

for the contribution of $F_{m+1}\left(\gamma_{n-2} \beta_{n-2}\right)$, since $F_{m} \vartheta_{x}=0$ for $x$ low.

By the lemma, we have

$$
\begin{aligned}
F_{m+1} \vartheta_{(-1, n)} & =F_{m} \vartheta_{(-1, n)}+(\lambda+\mu) F_{m} v_{n, 2(m-c)}+\lambda F_{m} v_{n, 2 m+1} \\
& \equiv(\mu+\lambda+\mu+\lambda) F_{m} v_{n, 2 m+1} \equiv 0 \quad \text { modulo } \Re^{4(m+1) r+2}
\end{aligned}
$$

and

$$
F_{m+1} \vartheta_{(-1, n-1)} \equiv F_{m} \vartheta_{(-1, n-1)}+\lambda F_{m} v_{n-1,2 m+1} \equiv F_{m} v \quad \text { modulo } \mathbb{R}^{4(m+1) r+2} .
$$

We will be done if we can show that

$$
F_{m+1} v \equiv F_{m} v \quad \text { modulo } \mathbb{R}^{4(m+1) r+2} .
$$

To see this, we first notice that we may replace $v$ by

$$
w=\left(\Psi^{-(2 c+1)} \gamma_{n-1}\right) l_{(2 c+1) r-1} \cdots l_{1} l_{0} \beta_{n-1},
$$

since both $F_{m}$ and $F_{m+1}$ vanish on $\vartheta_{x}$ for $x$ low. The path $w$ contains $\beta_{n-1}$ and $\Psi^{-s} \beta_{n-2}$ for $s=1, \ldots, 2 c+1$, but no other $\delta \in \Pi \beta_{j}$ for any $j$. Hence

$$
F_{m+1} v \equiv F_{m} v+(2 c+2)(\lambda+\mu) F_{m} v_{n-1,2 m+1}=F_{m} v \text { modulo } \Re^{4(m+1) r+2} .
$$

Notice that replacing an arrow in $w$ by a path of length $2(m-c) r+1$ yields a path of length $(2 m+1) r+2$, and the corrections arising from $u_{n-2}$ and $u_{n-1}$ are the only ones we have to take into account modulo $\Re^{4(m+1) r+2}$. 
ProOF OF THE Lemma. For $j=n-1$ or $j=n$ and for $x \leqslant y \leqslant x+n-2$, we define the two paths

$$
\begin{aligned}
& u(x, j, y):(x, j) \rightarrow(y, x+n-1-y) \rightarrow(y, k), \\
& w(x, j, y):(x, j) \rightarrow(y, x+n-1-y) \rightarrow\left(y, k^{\prime}\right),
\end{aligned}
$$

where $(y, k) \neq\left(y, k^{\prime}\right)$ are high and $(y, k)$ is congruent to $(x, j)$. Then $w(x, j, y)$ is a path of length $2(y-x)$ which lies in the ideal $\tilde{I}$ of (modified) mesh-relations, and therefore

$$
F_{m} w(x, j, y) \in \mathcal{R}^{2(y-x)+2(2 c+1) r}\left(\pi(x, j), \pi\left(y, k^{\prime}\right)\right) .
$$

In particular,

$$
F_{m} w(-1, j, t r) \equiv \kappa(j, t) F_{m} u(-1, j,(t+2 c+1) r) \quad \text { modulo } \Re^{2(t+2 c+1) r+2 r+2}
$$

for some $\kappa(j, t) \in k$. Since

$$
v_{j, t}= \begin{cases}u(-1, j, t r) & \text { for } t \text { odd } \\ w(-1, j, t r) & \text { for } t \text { even, }\end{cases}
$$

the proof of the lemma reduces to showing

$$
\begin{aligned}
\kappa(n-1,2 s) & = \begin{cases}0 & \text { for } s \text { odd } \\
1 & \text { for } s \text { even }\end{cases} \\
\kappa(n, 2 s) & = \begin{cases}1 & \text { for } s \text { odd } \\
0 & \text { for } s \text { even }\end{cases}
\end{aligned}
$$

We now compute $\kappa(j, t)$ by induction on $t$. For $t>0$, we obtain

$$
\begin{aligned}
F_{m} w(-1, j, t r)= & F_{m} u\left(t r-1, k^{\prime}, t r\right) F_{m} w(-1, j, t r-1) \\
& +F_{m} w(t r-1, k, t r) F_{m} u(-1, j, t r-1),
\end{aligned}
$$

where $\left\{k, k^{\prime}\right\}=\{n-1, n\}$ and $(t r-1, k)$ is congruent to $(-1, j)$. Indeed, since $F_{m}$ vanishes on all $\vartheta_{x}$ with $x$ low, we may first replace $w(-1, j, t r)$ by the low path $\delta l_{t r-1} w$ with

$$
w:(-1, j) \rightarrow(t r-1, n-1-t r) \rightarrow(t r-1, n-2)
$$

and then add $F_{m}\left(\delta \vartheta_{(t r-1, n-2)} w\right)$ to $F_{m} w(-1, j, t r)$.

In the first summand, we "push the crenel to the left" (3.2) until it contains a high vertex with first coordinate $(t-1) r$; only mesh-relations $\vartheta_{x}$ with $x \notin \Pi(-1, n) \cup$ $\Pi(-1, n)$ are necessary to do that. So the first summand satisfies

$$
\begin{aligned}
F_{m} u & \left(t r-1, k^{\prime}, t r\right) F_{m} w(-1, j, t r-1) \\
& =F_{m} u((t-1) r, q, t r) F_{m} w(-1, j,(t-1) r) \\
& \equiv \kappa(j, t-1) F_{m} u((t-1) r, q, t r) F_{m} u(-1, j,(t+2 c) r) \\
& \equiv \kappa(j, t-1) F_{m} u(-1, j,(t+2 c+1) r) \quad \operatorname{modulo} \mathcal{R}^{2(t+2 c+1)+2 r+2},
\end{aligned}
$$

where $((t-1) r, q)$ is the high vertex incongruent to $(-1, j)$.

As for the second summand, we notice that, modulo $\Re^{2(2 c+1) r+2 r+2}$,

$$
F_{m} w(t r-1, k, t r) \equiv\left\{\begin{array}{c}
F_{m} u(t r-1, k,(t+2 c+1) r) \\
\quad \text { for }(t r-1-k) \in \Pi(-1, n-1), \\
0 \quad \text { for }(t r-1, k) \in \Pi(-1, n) .
\end{array}\right.
$$


Adding up, we obtain

$$
\kappa(j, t)=\kappa(j, t-1)+ \begin{cases}1 & \text { if }(t r-1, k) \in \Pi(-1, n-1), \\ 0 & \text { if }(t r-1, k) \in \Pi(-1, n) .\end{cases}
$$

Remember that $(t r-1, k)$ is the high vertex with first coordinate $t r-1$ which is congruent to $(-1, j)$. If $t$ is even, $\Psi^{-t}(-1, j)$ is congruent to $(-1, j)$; if $t$ is odd it is not. We conclude that

$$
\begin{gathered}
\kappa(n-1, t)=\kappa(n-1, t-1)+ \begin{cases}0 & \text { if } t \text { is odd, } \\
1 & \text { if } t \text { is even, }\end{cases} \\
\kappa(n, t)=\kappa(n, t-1)+ \begin{cases}1 & \text { if } t \text { is odd } \\
0 & \text { if } t \text { is even. }\end{cases}
\end{gathered}
$$

These formulas, together with $\kappa(n-1,0)=1$ and $\kappa(n, 0)=0$ yield $(*)$ immediately.

3.9. Isomorphisms. We keep the assumptions of 3.8; i.e., $k$ has characteristic 2 and $N(\Gamma) \geqslant 0$. The last assertion we have to prove is that ${ }^{\prime} g_{0}=k \Gamma / J_{0}$ is not isomorphic to $\mathfrak{G}_{c}=k \Gamma / J_{c}$ for any $c>0$, nor to $k(\Gamma)$.

We denote the radical of any of these categories by $R$, and we note that for any $c>0, \Re_{c} / \mathcal{R}^{2 r+3}$ is isomorphic to $k(\Gamma) / R^{2 r+3}$, since the length of the path $v$ : $(-1, n-1) \rightarrow \Psi^{-(2 c+1)}(0, n-1)$ is $2(2 c+1) r+2 \geqslant 2 r+3$. Since isomorphisms preserve powers of the radical, it is therefore sufficient to prove that $h_{0} / \mathrm{C}^{2 r+3}$ is not isomorphic to $k(\Gamma) /{ }^{2} \Omega^{2 r+3}$.

Asume there is an isomorphism

$$
F: \mathscr{B}_{0} / \mathscr{Q}^{2 r+3} \rightarrow k(\Gamma) / \mathscr{Q}^{2 r+3} .
$$

We claim that the automorphism of the quiver $\Gamma$ induced by $F$ is compatible with $\tau$. Let $n \geqslant 5$ and assume that $F \pi(i, n-1)=\pi(p, n-1)$ for some $i, p \in \mathbf{Z}$. Then $F \pi(i, n-2)=\pi(p, n-2)$ and $F \pi(i-1, n-1)$ equals either $\pi(p-1, n-1)$ or $\pi(p-1, n)$. Since

$$
\mathscr{B}_{0} / \mathscr{Q}^{3}(\pi(i-1, n-1), \pi(i, n-1))=0
$$

and

$$
k(\Gamma) / R^{3}(\pi(p-1, n), \pi(p, n-1)) \neq 0,
$$

we see that $F \pi(i-1, n-1)=(p-1, n-1)$. Hence $F$ commutes with $\tau$ on the images under $\pi$ of high vertices of $\mathbf{Z} D_{n}$, and it clearly does on the other ones. The case $\mathbf{Z} D_{4}$ can be handled with an analogous argument. Since any automorphism of the translation-quiver $\Gamma$ induces an automorphism of $k(\Gamma) / \mathcal{O}^{2 r+3}$, we may assume that $F$ is the identity on $\Gamma$.

We obtain that for every arrow $\alpha$ of $\Gamma$,

$$
F \bar{\alpha} \equiv \lambda(\alpha) \bar{\alpha} \text { modulo } \text { Q }^{2 r+1},
$$

where $\lambda(\alpha) \in k \backslash\{0\}$ and where $\bar{\alpha}$ denotes the image of $\alpha$ in $\mathscr{G}_{0} / \mathscr{C}^{2 r+3}$ and

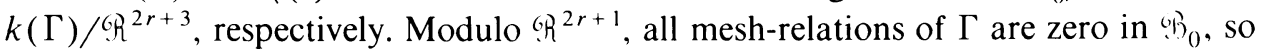
that

$$
\lambda(\alpha) \lambda(\sigma \alpha)=\lambda(\beta) \lambda(\sigma \beta)
$$


for any two paths $\alpha(\sigma \alpha), \beta(\sigma \beta)$ from any $x$ to $\tau^{-1} x$ in $\Gamma$. But then the functor sending $\bar{\alpha}$ to $\bar{\alpha} / \lambda(\alpha)$ is an automorphism of $k(\Gamma) / \mathcal{R}^{2 r+3}$, and hence we may assume that $\lambda(\alpha)=1$ for all $\alpha$.

The images of all paths in $\mathbf{Z} D_{n}$ of length $\geqslant 2 r+1$ which do not contain any high vertex generate an ideal $I$ in both $\mathscr{B}_{0} / \mathscr{C}^{2 r+3}$ and $k(\Gamma) / \mathcal{R}^{2 r+3}$, which is preserved by $F$. We let $\bar{F}$ be the induced functor modulo $I$. Observe that by 3.2

$$
\Re^{2 r+1} / \Re^{2 r+3}(x, y) \subseteq k(\Gamma) / \Re^{2 r+3}(x, y)
$$

is either zero or one-dimensional modulo $I$. In particular, if $x$ and $y$ are tail and head of an arrow $\alpha=\pi \alpha^{\prime}$, where $\alpha^{\prime}:(i, j) \rightarrow(p, q)$ is an arrow of $\mathbf{Z} D_{n}$, we obtain a one-dimensional space, say generated by $\bar{v}_{\alpha}$ for some path $v_{\alpha}$, if and only if $j \geqslant n-2-r$ and $q \geqslant n-2-r$. This condition is equivalent to saying that there is a path from $(i, j)$ to $\Psi^{-1}(p, q)$ in $\mathbf{Z} D_{n}$ containing a high vertex (compare 3.2). We obtain $\bar{F} \bar{\alpha}=\bar{\alpha}+\mu(\alpha) \overline{v_{\alpha}}$ for those arrows $\alpha$ and $\bar{F} \bar{\alpha}=\bar{\alpha}$ for the other ones. If we express the fact that the images of all $\vartheta_{x}$ with $x \notin \Pi(-1, n-1)$ are zero in $\mathscr{B}_{0}$, we obtain that $\Sigma \mu(\alpha)=0$, where $\alpha$ ranges over all arrows in the mesh starting at $\pi x$ for which $v_{\alpha}$ is defined. Since the image of $\vartheta_{(-1, n-1)}+v$ in $\mathscr{B}_{0}$ vanishes as well, we see that $\sum \mu(\alpha)+1=0$, where $\alpha$ runs through the arrows of the mesh starting at $\pi(-1, n-1)$. Notice that each $\mu(\alpha)$ arises exactly twice in this system of equations, once for each mesh containing $\alpha$. We obtain a contradiction by adding up all the equations, since $k$ has characteristic 2 .

\section{REFERENCES}

1. M. Auslander, Representation theory of Artin algebras. II, Comm. Algebra 1 (1974), 269-310.

2. K. Bongartz and P. Gabriel, Covering spaces in representation-theory, Invent. Math. 65 (1982), 331-378.

3. P. Gabriel, Auslander-Reiten sequences and representation-finite algebras, Representation Theory I, Lecture Notes in Math., vol. 831, Springer-Verlag, Berlin and New York, 1980, pp. 1-71.

4. Ch. Riedtmann, Algebren, Darstellungsköcher, Überlagerungen und zurück, Comment. Math. Helv. 55 (1980), 199-224.

5. __ Representation-finite selfinjective algebras of class $A_{n}$, Representation Theory II, Lecture Notes in Math., vol. 832, Springer-Verlag, Berlin and New York, 1980, pp. 449-520.

6. Configurations of $\mathbf{Z} D_{n}$, J. Algebra 82 (1983), 309-327.

7. _ Representation-finite selfinjective algebras of class $D_{n}$, Compositio Math. 49 (1983), 231-282.

8. Many algebras with the same Auslander-Reiten quiver, Bull. London Math. Soc. 15 (1983), $43-47$

9. A. Roiter, Generalization of Bongartz' theorem, Mathematics Inst. Ukrainian Akad. of Sciences, Kiev, 1981, pp. 1-32.

Department of Mathematics, Mathematisches Institut, Rheinsprung 21, CH-4051 Basel, SWITZERLAND

Current address: Institut Fourier, Université de Grenoble, B. P. 116, F-38402 St. Martin d'Hères, Cedex France 